or its open access mirror

Elsevier Editorial system(tm) for Nutrition

Manuscript Draft

Manuscript Number: NUT-D-19-00533R1

Title: Health status and nutritional development of adopted Ethiopian children living in Southern spain: A prospective cohort study

Article Type: Original Investigation

Keywords: health status; adopted children; Ethiopia; growth; Mediterranean diet; KIDMED.

Corresponding Author: Dr. Juan José Hernández Morante, Ph.D.

Corresponding Author's Institution: Catholic University of Murcia

First Author: Juan José Hernández Morante, Ph.D.

Order of Authors: Juan José Hernández Morante, Ph.D.; Carmen Piernas Sánchez, Ph.D.; Daniel Guillén Martínez, Ph.D.; Antonio Pardo-Caballero, R.N.; María José Fernández Abellán, R.N.; Isabel Morales Moreno, Ph.D.

Abstract: Objective: To evaluate the health status and anthropometrical development of adopted children from Ethiopia living in southern Spain. A second objective was to evaluate the association between these parameters and the adherence to the Mediterranean dietary pattern.

Methods: 53 families with adopted children agreed to participate. Similarly, 54 native-born children were selected. A physical examination of the children, including height and weight, was conducted in Ethiopia at the time of entry in the adoption process. Height and weight were remeasured at the first day of adoption and 6, 12 and 24-month postadoption. After 2 years of follow-up, a new physical examination, including the KIDMED test to measure adherence to Mediterranean diet, was conducted.

Results: Skin and digestive conditions were the most prevalent disorders in Ethiopian children before adoption and at the end of follow-up. Baseline anthropometric characteristics indicated a low wasting prevalence (7.5\%); however, stunted growth was more evident (35.8\%). After 6 months, the weight-for-age (WAZ) of these children was restored (change from baseline $\mathrm{p}<0.001$ ), and 1-year post-adoption the data were similar to those of Spanish children (p>0.050). Although height-for-age (HAZ) also increased from baseline $(\mathrm{p}<0.001)$, the effect of adoption was not as evident. The KIDMED score was associated with greater WAZ $(r=0.279 ; \mathrm{p}=0.045)$ and HAZ $(r=0.385 ; \mathrm{p}=0.004)$.

Conclusions:

This prospective study of adopted Ethiopian children confirmed a rapid growth development which occurred from the beginning of the adoption process and continued after the 24-month follow-up. A higher adherence to the Mediterranean diet was associated with better growth development, which reinforces the importance of a balanced and adequate diet in growing children. 


\author{
Juan José Hernández Morante \\ Faculty of Nursing. \\ Catholic University of Murcia \\ 30107 Murcia, Spain \\ E-mail: jjhernandez@ucam.edu \\ Phone: +34 968278543 \\ Fax: +34 968278649
}

Monday, August 12, 2019

To the Editorial Office:

Dear Editor,

We are pleased to submit the revised version of our manuscript entitled \# NUT-D-1900533 "Health status and nutritional development of adopted Ethiopian children living in Southern Spain: A prospective cohort study", on behalf of all my co-authors, for publication in Nutrition. For a better understanding of the modifications made in response to the reviewer's comments, the changes performed in the revised paper have been highlighted in yellow. The language mistakes corrected have not been highlighted, with the aim of to identify those changes performed attending to the reviewers' recommendations.

Therefore, we believe the manuscript is much strengthened in its present form.

Yours sincerely.

Dr. Juan José Hernández Morante. 
Ms. Ref. No.: NUT-D-19-00533

Title: Health status and nutritional development of adopted Ethiopian children living in Southern Spain: A prospective cohort study

Reviewers' comments:

Reviewer \#1:

At the outset, my compliments on an original research article, a prospective study that evaluated the nutritional status progression of the adopted Ethiopian children. Results are encouraging and interesting - it has the potential to become a multi-centric study. Following are few of my observations and suggestions-

\#1.- 1. Title: The term Acculturation could be removed from the title. It appears only in the title and then as a keyword. There is no explanation / connect in the article context either in background or discussion.

We agree with the reviewer's comment, using the term "acculturation" would require a much deeper background on this and our research is mostly focused on nutrition and dietary changes, without looking into any social aspects. Therefore, we have changed the title and the keyword in the revised paper.

\#2.- 2. Study design and methodology is explained well. *In addition to Anthropometric characteristics, assessment of health status and adherence to Mediterranean dietary pattern (KIDMED test) should also have been done for the matched sample of children from Murcia (Spain). This would have helped to answer some questions and abjectly compare the two groups by testing for any significant differences. In hindsight, this can at least be mentioned in the limitations section or suggested for further research.

It is indeed a limitation that the KIDMED test and health status was not performed in the matched sample. We have included a statement in the discussion section with regards to this.

\#3.- *The Anthropometric data was not personally recorded by the research team; it was reported by the parents from the children's growth charts. Please explain how it was ensured that staff at the Community Health Centres were trained in standard techniques of taking and recording anthropometric measurements and equipment used for the same was standardized / validated.

We apologise if this information was not clearly stated in the original paper. The children's growth chart was filled by the staff, not by the parents. The parents just provided the data recorded in these growth-chart reports. We have stated this more clearly in the revised paper.

The nurse staff of the Community Health Centres in Spain is specialized in paediatric care. Namely, they are not general nurses but paediatric nurses, and they receive extensive training on children anthropometric evaluation as a part of their specialization. In addition, data collection is performed at specific times for the entire population as part of the Spanish health system prevention initiative. 
\#4.- 3. Results: They address all the objectives. ${ }^{*}$ The data is not normally distributed (maybe because of small $N$ ), as is evident by the mean and standard deviation values in Table 1 for Age, WAZ, HAZ BAZ and WHZ. So it should be represented as non-parametric data with median and IQR values (median [Q1, Q3]). Please consult a statistician for appropriate non-parametric tests for analysing your data (including Repeated Measures ANOVA).

We agree with the reviewer and have included this data in table 1 as suggested. In addition, we have consulted the Department of Biostatistics of the University about the use of non-parametric testing. They noted that that the growth data is z-standardized, (also known as normalized) meaning it meets the normality assumption of the ANOVA test performed with this data. To confirm this and taking into account the population size, the Shapiro-Wilk test was performed, confirming the normal distribution of standardized data.

\#5.- *Please add data pertaining to the Mediterranean diet adherence score (low, average, good) categories after Table 2.

We have included the information in the revised paper.

\#6.- *For your second objective, to evaluate the association between growth parameters and adherence to the Mediterranean dietary pattern, you have done a correlation. So, please write your results accordingly. Mention it as significant positive correlation and also state whether the correlation was weak, moderate or strong and it's implications.

Thank you for this remark. We have modified this paragraph in the Results section to clarify this.

\#7.- Discussion: Well written and appropriate in context. Your study found that certain health problems increased after the adoption process like dental, visual and some new ones detected like cardiovascular or respiratory problems two years in Spain. One possible reason that could explain this would be intrauterine growth retardation (IUGD) and developmental origin of disease owing to fetal re-programming. You could explore literature on this and add in your discussion.

We appreciate the reviewer's comments. This last suggestion highlighted a very relevant topic; therefore, we have included a sentence in the discussion section of the revised paper about this. In addition, two new references have been included to clarify these aspects [1,2]: 
Reviewer \#2:

Thank you for giving me the opportunity to comment on this interesting study. It presents both sociological and nutritional aspects. The authors should appraised for bringing out new results on a such highly interested and timely topic. The study however manifests several methodological issues.

\section{Major comments}

I would characterize the sociological as the most interesting and original part of this study. My main concern is regarding the causative relationship of the adherence to MED diet to the overall growth of these children. Children's growth does not solely depends on nutritional status, but also on a stable, safe and caring environment. At that basis, it would be prudent to compare children from an orphanage from the same region, since lifestyle habits and conditions, would significantly affect the growth development of any child. These parameters might not be incorporated in any analysis, however, might profoundly affect study findings. So, my first main concern would be on the initial design of the study: 1) the significant psychological effects of living in an orphanage would be partially "excluded", if the authors tried to focus on children with a similar domestic background in Spain. This might affect the study results.

We appreciate the reviewer's comments. Effectively, children's growth does not solely depend on nutritional status, but also on other environmental and psychological factors. Undoubtedly, it would have been very interesting to compare children from an orphanage from the same region, but this was not originally planned in the early stages of the cohort which was exclusively focused on Ethiopian children. Overall, our study design is consistent with previous studies performed in similar populations [3,4], which have not included adopted children from an orphanage from the same region.

Nevertheless, we absolutely agree with the reviewer and regret the lack of information on this important topic. We have found two new references explaining the influence of psychological and sociological issues on Ethiopian children development which have been included in the discussion section [5,6]. For example, Worku et al. have described that Ethiopian children living in extreme poverty performed worse in all developmental domains [6], which suggest that the adoption process may influence not only anthropometrical parameters but also neuropsychological development.

2) My second comment would on the neurocognitive and emotional development of these children compared to Spanish children. I would be very keen to see the effects of a stable living background on the catch-up growth of Ethiopian chlidren. Unfortunatelly, the major aspect of the emotional, cognitive and psychological profile has not been included in the study and might alone explain, improvements in the somatic growth. Overall, the authors might be interested in providing a global perspective of the health of these children, as defined by WHO, including both the mental as well as the somatic aspect.

We fully agree with the reviewer comments. In the present work, we have been focused on health status and anthropometrical evolution, but undoubtedly, the relevance of emotional, cognitive and psychological factors is of great interest to us and will keep this in mind for future measurements. As noted above, previous research suggest that the adoption process can improve neurological development of adopted children, and it would be interesting to investigate this in our cohort. We have included 
this as a limitation, and we have also modified several paragraphs in the Discussion section to comment these aspects.

3) My main concern is regarding the author's hypothesis of an association of the adherence to MED diet and catch-up growth. According Table 2, only 64\% of these children reported adherence to MED diet. The authors reported a small, but significant association between KIDMED and increases in WHZ. However, a similar analysis for children not adhered to MED diet was not conducted. As the authors MED diet is associated with better growth development, but this conclusion does not apply for $64 \%$ of the children included in the study. A causative effect cannot be established and I would suggest revising the main conclusions (secondary) of this study. In my perspective, several other factors (as I mentioned earlier ) might be related to the improvement in growth, but such associations were not evident in the study.

We apologise for the lack of clarity in this regard. The correlation analysis was performed in all the adopted children (100\% of the sample studied), and our data indicates that those with better adherence to Mediterranean diet showed better anthropometrical evolution. We have modified this section of the Results to clarify this aspect. We have also revised our language throughout to avoid implying causative effects and better reflect our actual observations. As mentioned above, further limitations have been added to highlight the lack of data on other important factors which may contribute to explain the growth improvements.

\section{Minor points}

-Several language errors are present throughout the text.

We apologize for any grammatical errors. We have carefully checked the text throughout to make sure the language is appropriate.

-Please remove the tables in the end of the text.

We have tried to adhere to the journal's instructions to include the tables at the end of the relevant paragraph.

-Were Ethiopian children from the same geographical region?

Yes, all children were from Addis Ababa city.

-Are there any results on macro and micronutrient intakes regarding their stay in Ethiopia and Spain? Was a dietary analysis conducted?

Unfortunately, we did not collect this type of data. Initially we tried to collect this information, but most of the parents refused to perform the questionnaires. Given this was unfeasible, we used the KIDMED questionnaire as a surrogate measure of dietary quality.

-Is there any educational activity profile available for these children?

To our knowledge, there is no specific educational activity profile for these children and are usually included in the same education program that Spanish children. 


\section{-I would suggest reporting main children pathologies, not just as skin disorders etc.}

We appreciate the reviewer comment. We initially discussed different ways to report this but given the broad range of conditions described in this population, we considered it was less confusing to report it the way we have done which in our opinion facilitates the interpretation of the children health status. However, we could detail all the different diseases if the reviewer and editor consider this pertinent.

\section{-I am not clear (p.7 line 154,154) about the authors statement "several medical problems were detected after 2 years of adoption"}

We apologize for this misunderstanding. We were referring that several medical diseases not detected at baseline were detected or appeared after 2 years of adoption.

Finally, we would like to agree the reviewers for their comments. We sincerely believe that with the modifications suggested, the paper quality has improved significantly.

\section{REFERENCES:}

[1] Zou K, Ding G, Huang H. Advances in research into gamete and embryo-fetal origins of adult diseases. Sci China Life Sci 2019;62:360-8. doi:10.1007/s11427-018-9427-4.

[2] Gapp K, von Ziegler L, Tweedie-Cullen RY, Mansuy IM. Early life epigenetic programming and transmission of stress-induced traits in mammals: How and when can environmental factors influence traits and their transgenerational inheritance? BioEssays 2014. doi:10.1002/bies.201300116.

[3] Fuglestad AJ, Kroupina MG, Johnson DE, Georgieff MK. Micronutrient status and neurodevelopment in internationally adopted children. Acta Paediatr Int $\mathrm{J}$ Paediatr 2016;105:e67-76. doi:10.1111/apa.13234.

[4] Miller LC, Tseng B, Tirella LG, Chan W, Feig E. Health of children adopted from Ethiopia. Matern Child Health J 2008;12:599-605. doi:10.1007/s10995-007-0274-4.

[5] Servili C, Medhin G, Hanlon C, Tomlinson M, Worku B, Baheretibeb Y, et al. Maternal common mental disorders and infant development in Ethiopia: The P-MaMiE Birth Cohort. BMC Public Health 2010;10. doi:10.1186/1471-2458-10-693.

[6] Worku BN, Abessa TG, Wondafrash M, Vanvuchelen M, Bruckers L, Kolsteren P, et al. The relationship of undernutrition/psychosocial factors and developmental outcomes of children in extreme poverty in Ethiopia. BMC Pediatr 2018;18:1-9. doi:10.1186/s12887018-1009-y. 


\section{ACCULTURATION AND NUTRITIONAL DEVELOPMENT OF ADOPTED ETHIOPIAN CHILDREN LIVING IN SOUTHERN SPAIN: A PROSPECTIVE COHORT STUDY}

Running head: Nutritional evolution of adopted Ethiopians

Juan José Hernández-Morante ${ }^{\mathrm{a},{ }^{*}}$, Ph.D., Carmen Piernas-Sánchez ${ }^{\mathrm{b}}$, Ph.D., Daniel

Guillén-Martínez ${ }^{\mathrm{a}}$, Ph.D., Antonio Pardo-Caballero ${ }^{c}$, R.N., María José FernándezAbellán $^{\mathrm{d}}$, R.N., Isabel Morales-Moreno ${ }^{\mathrm{a}}$, Ph.D.

${ }^{\mathrm{a}}$ Faculty of Nursing, Catholic University of Murcia (Murcia), Spain

${ }^{\mathrm{b}}$ Nuffield Department of Primary Care Health Sciences, University of Oxford, United Kingdom

c University Hospital "Santa Lucía” of Cartagena (Murcia), Spain.

dPrimary Health Care Center "El Carmen” of Murcia (Murcia), Spain.

\section{*Corresponding Author:}

Dr. Juan José Hernández Morante.

Campus de Guadalupe, Avda de Los Jerónimos, s/n, 30107, Murcia, Spain.

Phone: +34 968278 543; E-mail: jjhernandez@ucam.edu

Word Count: 4844 Number of figures: 5 Number of tables: 2

Authorship: D.G.M., M.J.F.A. and A.P.C. contributed to acquisition, analysis, and interpretation of the data. I.M.M., C.P.S. and J.J.H.M. contributed to conception and design, contributed to analysis, and interpretation of the data, drafted manuscript, critically revised manuscript and gave final approval.

\section{Declaration of Interest statement:}

The authors have no conflict of interest to declare. 


\section{HIGHLIGHTS}

- Adopted Ethiopian children show clinical conditions and chronic undernutrition

- There are few prospective studies evaluating the evolution of adopted children

- Skin and digestive conditions were very prevalent in Ethiopian children before and post-adoption

- A fast anthropometric growth was observed the 24-month follow-up

- Adherence to Mediterranean diet was related to better growth of these children. 
1 Health status and nutritional development of adopted Ethiopian children living in

\section{Southern Spain: A prospective cohort study}

\section{Abstract}

4 5

Objective: To evaluate the health status and anthropometrical development of adopted children from Ethiopia living in southern Spain. A second objective was to evaluate the association between these parameters and the adherence to the Mediterranean dietary pattern.

Methods: The study sample included 53 adopted children from Ethiopia and a matched sample of 54 native-born children. A physical examination of the children, including height and weight, was conducted in Ethiopia at the time of entry in the adoption process. Height and weight were re-measured at the first day of adoption and 6, 12 and 24-month post-adoption. After 2 years of follow-up, another physical examination was performed, including the KIDMED test to measure adherence to Mediterranean diet.

Results: Skin and digestive conditions were the most prevalent disorders in Ethiopian children before adoption and at the end of follow-up. Baseline anthropometric characteristics indicated a low wasting prevalence (7.5\%); however, stunted growth was more prevalent $(35.8 \%)$. After 6 months, the weight-for-age (WAZ) of Ethiopian children was restored (change from baseline $p<0.001$ ), and not significantly different from the Spanish children at 1-year post-adoption. Height-for-age (HAZ) also increased from baseline $(p<0.001$. A higher KIDMED score was associated with increased WAZ $(\mathrm{r}=0.279 ; p=0.045)$ and HAZ $(\mathrm{r}=0.385 ; p=0.004)$.

\section{Conclusions:}

This prospective study of adopted Ethiopian children confirmed a rapid growth development which occurred from the beginning of the adoption process and continued after the 2-years of follow-up. A higher adherence to the Mediterranean diet was associated with better growth development, which reinforces the importance of a balanced and adequate diet in growing children.

Keywords: health status, adopted children, Ethiopia, growth, Mediterranean diet, KIDMED. 
Adoption of children from developing countries has increased over time, becoming an important social phenomenon. From a health perspective, children adopted from developing countries are considered at higher risk, since the incidence of health problems is significantly higher than in native-born children [1]. Among the main health problems detected in these children, neurological development, physical growth and nutritional disorders are the most frequent, although anemia, infectious diseases, acute respiratory infections and intestinal parasitosis are also common [2-4].

Adoption is a turning point in the evolutionary development of children; it means the beginning of a period of stability in 'optimal' psychological and physiological conditions for their development [5]. Three kinds of risk factors for the proper development of adopted children have been identified: the birthplace, the risk factors before institutionalization, and the risk factors that appear during institutionalization[6]. However, very few studies have examined the consequences of adoption and the subsequent health and nutritional evolution of these children.

Previous studies which focused on the health status of adopted children have highlighted significant growth delays at the time of arrival in their adoptive families $[7,8]$. Ethiopian children, who are most frequently adopted in Spain, have been studied in a few previous prospective studies, but none have been performed in our environment. Demographic data from Ethiopia have described that 29\% of Ethiopian children have moderate to severe underweight, and $44 \%$ showed lower height. In addition, malnutrition has been described as responsible for $54 \%$ of infant mortality [9], which suggests that adopted children from Ethiopia may be at a higher risk of nutritional disorders. A previous study have also described the health status and development of adopted children from Ethiopia, showing that these children had better growth compared to adopted children from China, Guatemala or Russia [6]. The authors speculated that children from Ethiopia spend less time in institutional care and more time with their biological families, although there may be other differences in terms of the quality of institutional care or other growth differences of ethnic nature [6].

One the most striking changes for Ethiopian children may be related to the adoption of the Mediterranean dietary pattern[10]. Although the traditional Mediterranean diet has been associated with many health benefits, the evolution of food 
63 consumption in Mediterranean countries is currently not encouraging as the population 64 moves towards a higher intake of energy-dense nutrient-poor foods [11]. However, 65 there are no previous investigations of the adherence of Ethiopian children to a 66 Mediterranean dietary pattern after adoption, neither on the association between 67 Mediterranean diet and developmental growth of these children. This study aims to 68 examine the health status and anthropometrical development of Ethiopian children after 692 years living in southern Spain. A secondary objective is to evaluate the association 70 between adherence to the Mediterranean diet and the anthropometrical development of 71 these children. 
72 SUBJECTS AND METHODS

73

74

75

76

77

78

79

80

81

82

83

21

22

23

24

25

26

27

28

29

30

31

32

33

34

35

36

37

38

39

40

41

42

43

44

45

46

47

48

49

50

51

52

53

54

55

56

57

58

59

60

61

62

63

64

65

\section{Design and Subjects}

All Ethiopian children adopted in Murcia (Spain) during the period of January 2010 to December 2016 were eligible and invited to take part in the cohort $(n=336$ children). Families were contacted through e-mail or telephone, and 176 families volunteered to take part in the study (52.4\% response rate) and 53 completed all the baseline evaluations. During the same period, a matched sample of 57 children from Murcia (Spain) (31 girls and 26 boys of the same age), were also recruited for the purpose of comparison between the sample of adopted Ethiopian children and the native-born children. The sample of native-born children was obtained through collaboration with two schools in Murcia. Figure 1 describe the flow diagram of the study.

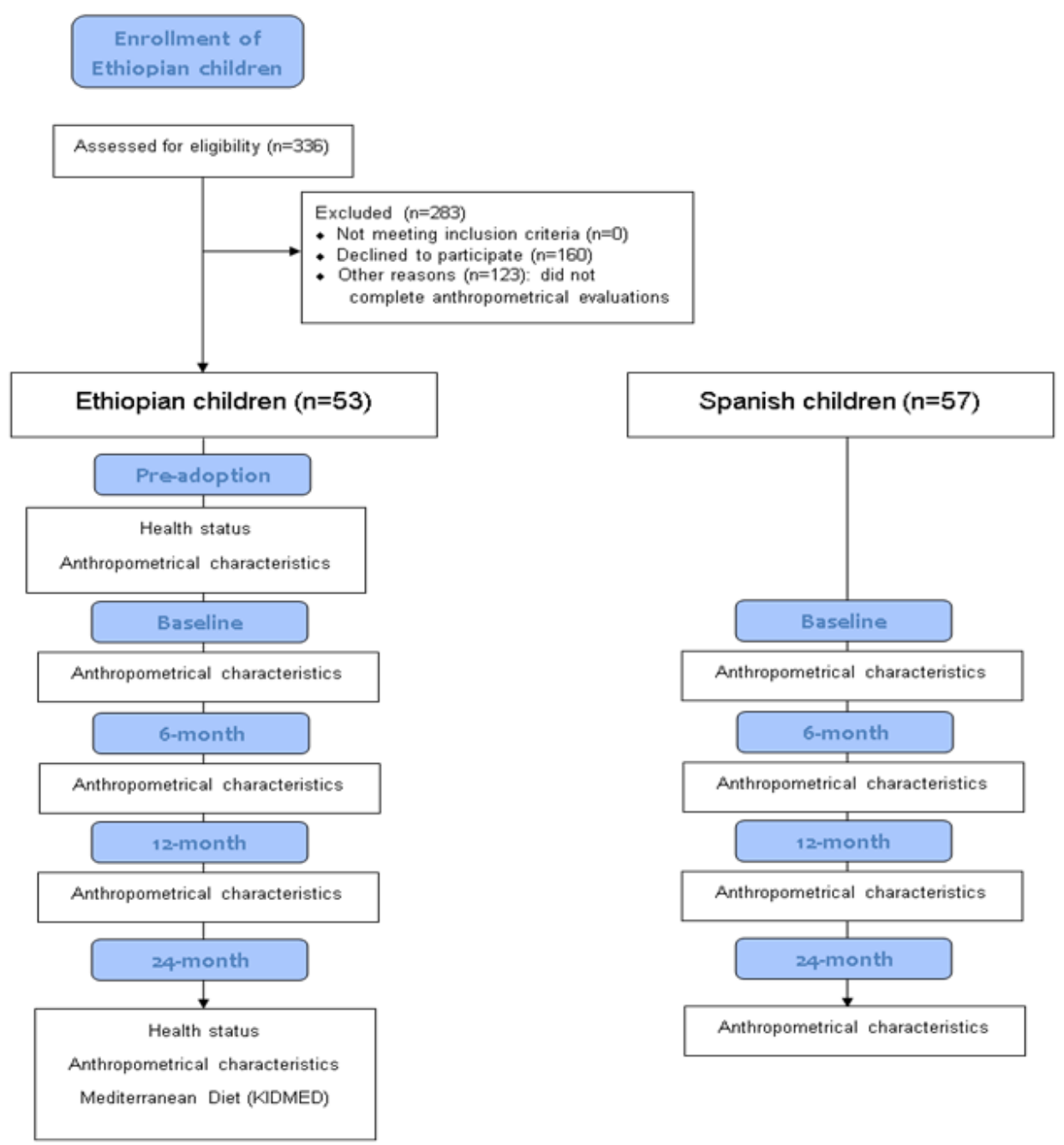

Figure 1. Flow diagram of the present study. 
87 The study was carried out after receiving written authorization from the Collaborative 88 Entities of International Adoption - AMOFREM (Murcia, Spain). The Ethics

89

90

91

92

93

94 Committee of this Association approved the study. The data obtained in the present work was stored in this association. Parents were informed orally and in writing about the study. They were also given an explanation of the ethical aspects of the project, informing the possible participants about the main objective of the study and guaranteeing the confidentiality and anonymity of the data, in accordance with the Declaration of Helsinki and Biomedical Research Spanish Laws. All parents of Ethiopian and Spanish children provided written informed consent.

\section{Measurements}

A physical examination of the adopted children was conducted at the time they entered the orphanage in Ethiopia (Pre-adoption period). This data was accessed through the AMOFREM (Asociacion Motivacion Familia Y Recursos Matrimoniales) association. This physical examination evaluated the presence/absence of visual, hearing, cardiovascular, respiratory, digestive, cutaneous, reproductive, cognitive, psychomotor, infectious and dental problems or any other clinical condition of interest. Other socio-demographic data, such as age (as reported by the Ethiopian adoption centre), sex and stay time at the institution/orphanage were recorded. The same physical examination was performed at the end of the follow-up period (2-year post-adoption).

Height and weight measurements were taken before adoption at the time of arrival in Murcia (baseline) and at 6 months, 12 months and 2 years later. The same measurements were obtained from both Ethiopian and Spanish children following the WHO guidelines for the evaluation of children growth [12]. BMI was calculated as the weight in kilograms divided by the squared height in meters. All measurements were zstandardized according to the WHO Child Growth standards. To better interpret the growth pattern of Ethiopian adopted children, height-for-age, weight-for-age and BMIfor-age parameters were calculated using the WHO-Anthro 3.0 Software [13]. Measurements were performed in the respective Community Health Centres and recorded in a growth-chart report by the paediatric nurse staff, from which parents reported the required study data. 
At 2 years of follow-up, Ethiopian children completed the KIDMED test to evaluate their adherence to the Mediterranean dietary pattern[14]. This validated test is composed by 16 items, divided in 12 items which are considered healthy habits (e.g. higher consumption of oil, fish, fruits, vegetables, cereals, nuts, pulses, pasta or rice, dairy products, and yoghurt), and 4 items related to unhealthier habits (e.g. higher consumption of fast food, baked goods, sweets, and skipping breakfast). The presence of healthier habits was scored as +1 , while negative habits were scored as -1 . The scoring range of the test is $0-12$ points, where $0-3$ points indicates a low adherence to the Mediterranean diet, a score of 4-7 describes average adherence, and a score of 8-12 reflects good adherence to the Mediterranean dietary pattern [14]. Previous data have described a moderate to excellent reliability of this test $(\kappa=0.6-0.9)[15]$.

\section{Statistical Analysis}

A frequency analysis of categorical variables, such as the presence of diseases, was estimated at the beginning and at the end of the follow-up period. McNemar test was employed to determine whether the proportion of children who had a clinical condition before adoption decreased after 2 years of follow-up. In order to analyse mean differences of anthropometrical parameters over the different follow-up times (baseline in Ethiopia, arrival, 6 months, 12 months and 2 years), a repeated-measures ANOVA was conducted with a post hoc test of Sidak correction, using time as the within-subjects factor, and group (sex and origin) as the between-subjects factors. The same procedure was conducted to compare progression of Ethiopian and Spanish children. In order to investigate the association between the KIDMED test score and the children's growth, a correlation analysis adjusting for children's age and sex was performed. The data were analysed using SPSS 24.0 for Windows, and a $p<0.05$ was set to denote statistical significance. 


\section{RESULTS}

146

147

148

149

150

151

152

153

154

155

156

\section{General characteristics and health status}

Data from the pre-adoption report showed that the mean age of children entering the orphanage in Ethiopia was 23 months, while the age at the time of adoption was 30 months. There were no statistical differences in age between Ethiopian girls and boys neither at the time of entry into the orphanage $(p=0.160)$ or at their arrival to Murcia $(p=0.292)$.

Baseline anthropometric characteristics of Ethiopian children indicated a general malnutrition status, since most parameters (adjusted to age) showed negative z-scores (Table 1). Baseline wasting or thinness prevalence, considered as z-weight-forlength/height $(\mathrm{WHZ})<-2$ was of $7.5 \%$; while baseline stunted growth, determined as zheight-for-age $(\mathrm{HAZ})<-2$ was more prevalent $(35.8 \%)$.

Ethiopian children $(\mathbf{n}=\mathbf{5 3})$

\begin{tabular}{l|c}
\hline \begin{tabular}{l} 
Sex: Female \\
\multicolumn{1}{c|}{ Male }
\end{tabular} & $18(34 \%)$ \\
\hline Age $(\mathrm{mo})$ & $35(66 \%)$ \\
\hline Weight & $21(6$ to 36$)$ \\
\hline z-weight-for-age & $10.5(7.0$ to 15.0$)$ \\
\hline Height $(\mathrm{cm})$ & $-0.91(-1.89$ to -0.10$)$ \\
\hline z-height-for-age & $80.0(63.0$ to 94.0$)$ \\
\hline BMI & $-1.16(-2.76$ to -0.13$)$ \\
\hline z-bmi-for-age & $16.10(14.70$ to 17.90$)$ \\
\hline z-weight-for-height & $0.20(-0.93$ to 0.90$)$ \\
\hline
\end{tabular}

157 Table 1. Baseline characteristics of Ethiopian children. Data represent median 158 with interquartile range (Q1-Q3). BMI: Body mass index.

The main health problems of the adopted Ethiopian children before adoption were related to skin, digestive and psychomotor alterations (Figure 2). Similarly, skin 162 and digestive alterations remained as the most prevalent disorders 2 years after 163 adoption, although the number of cases was significantly reduced. Importantly, several 164 medical problems not observed at baseline, including visual, cardiovascular and reproductive disorders, were detected or appeared 2 years after adoption (Figure 2). 


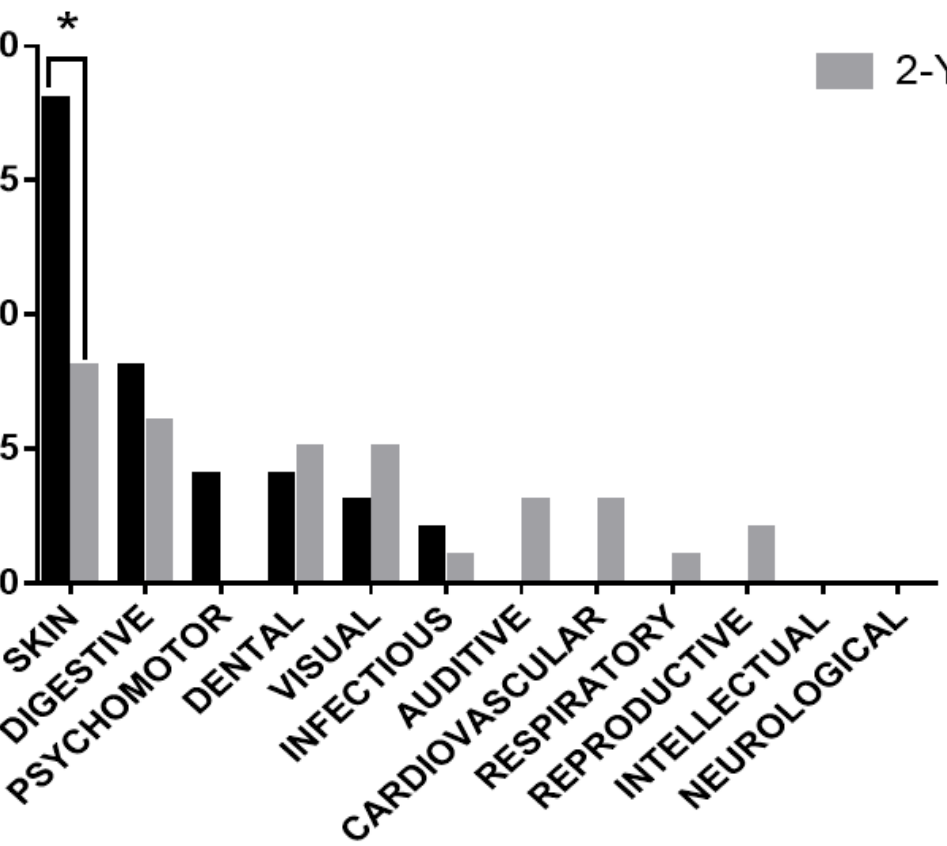

\section{Evolution of anthropometrical parameters}

Among Ethiopian boys, repeated measures ANOVA revealed a significant effect of time on the different parameters evaluated (Figure 3). Baseline z-scores of z-weightfor-age (WAZ) and HAZ indicated a delay on children's growth (Fig. 3A and 3B). At baseline, HAZ values were significantly lower in Ethiopian children than in the comparator group of Spanish children. However, these differences disappeared at 1 year and HAZ values were not different at 2 years of follow-up (Fig. 3B). Other indicators such as z-bmi-for-age (BAZ) and WHZ showed a similar trend for both Ethiopian and Spanish children (Fig. 3C and 3D). 
A

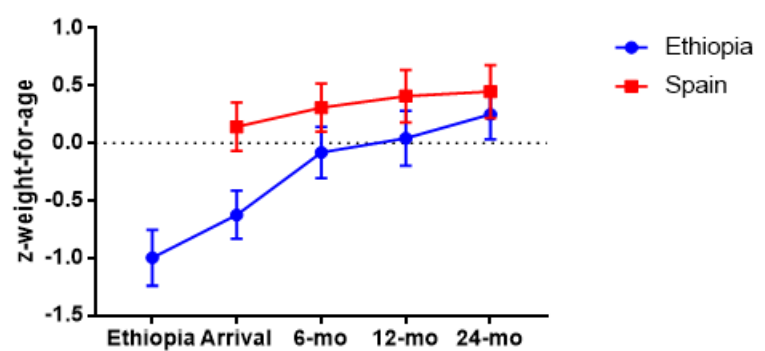

C

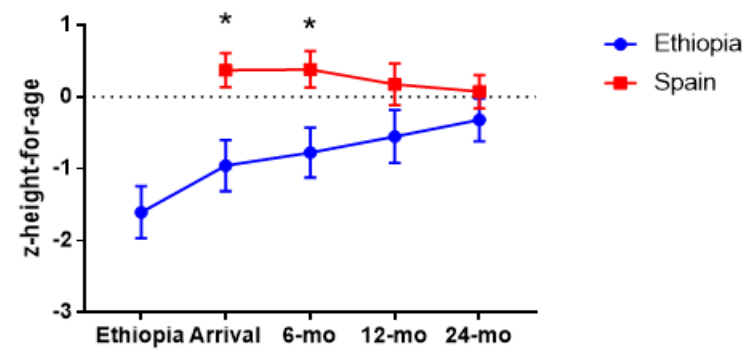

B

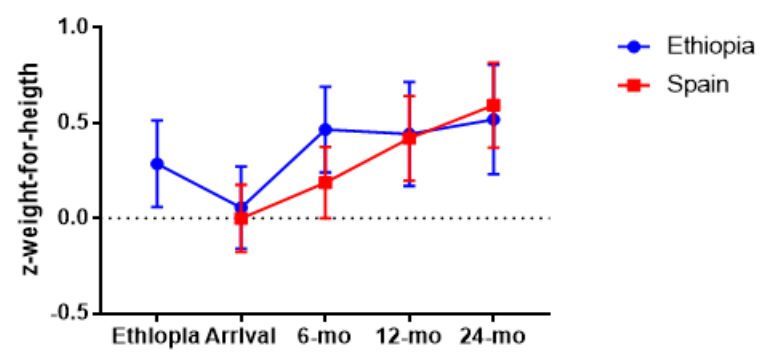

D

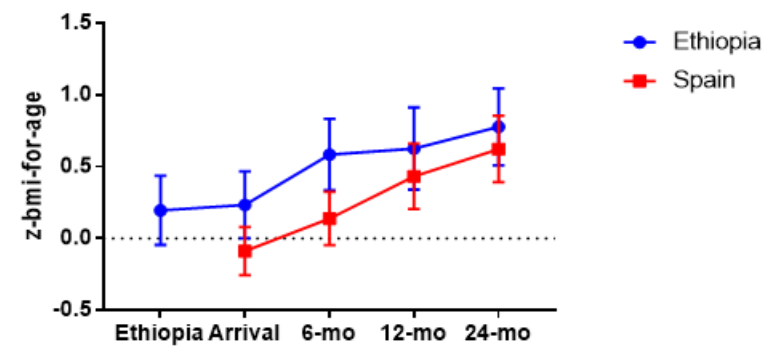

Figure 3. Evolution of the anthropometrical parameters of the Ethiopian boys compared to the Spanish boys. Data indicate mean \pm sd. Baseline and follow-up z-scores for z-weightfor-age (A), z-weight-for-height (B), z-height-for-age (C) and z-bmi-for-age (D) are shown. The dotted line represents a $\mathrm{z}$-score $=\mathbf{0}$. Repeated measures ANOVA showed a statistically significant effect of time $(p<0.001)$ in all parameters. Sidak's multiple comparisons test was performed to compare data between Ethiopian and Spanish boys. $* p<0.050$. 
A

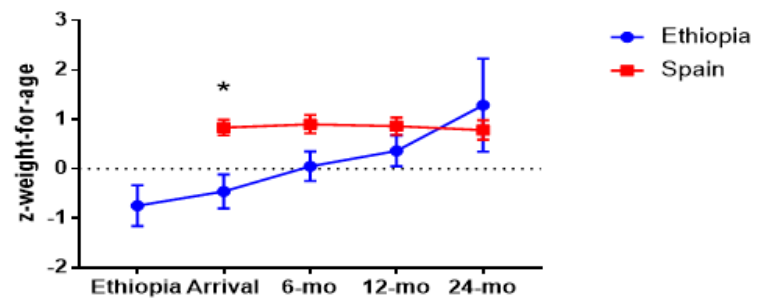

C

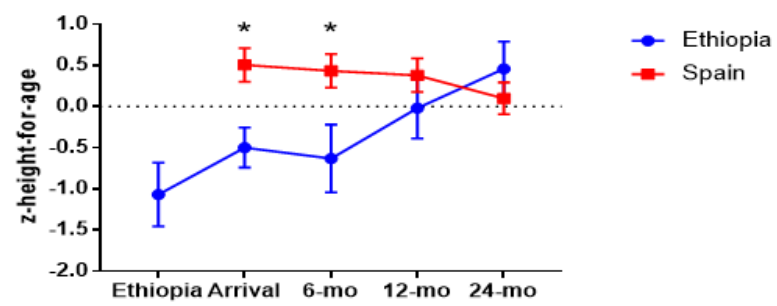

B

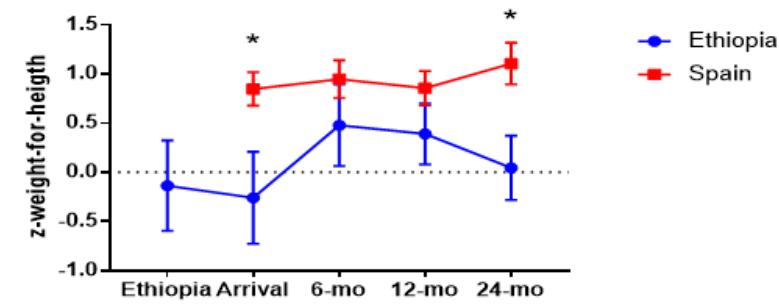

D

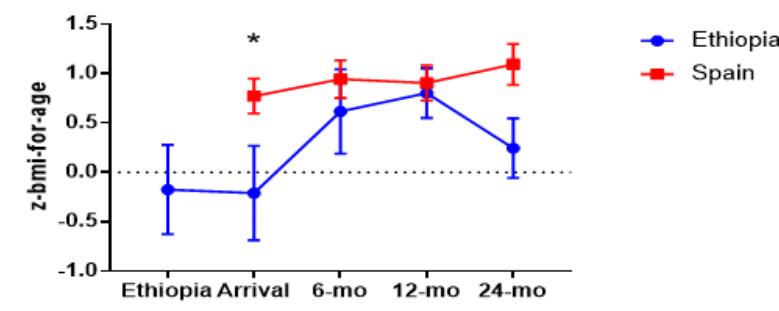

When the evolution of anthropometric parameters among Ethiopian boys and girls was compared (Figure 5), our data revealed that, although both improved significantly, WHZ and BAZ data were higher in boys after 24 months (Fig. 5C and 5D), while Ethiopian girls showed higher HAZ, especially after 12 and 24-month postarrival (Fig. 5B). In fact, Ethiopian girls acquired a normalized height after 12 months; however, Ethiopian boys were not able to reach an average height $(\mathrm{HAZ}=0)$, even after 24-month post-adoption. This situation was probably due to the higher baseline height of Ethiopian girls, although our data did not reflect a statistical difference in baseline HAZ between boys and girls $(p=0.204)$. 
A

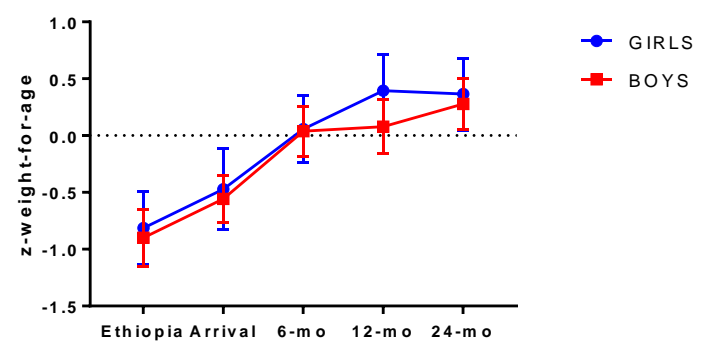

C

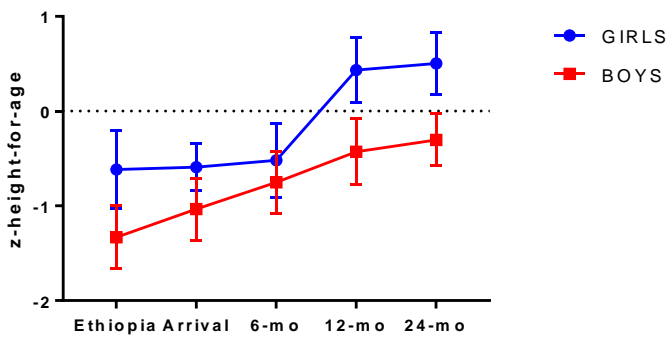

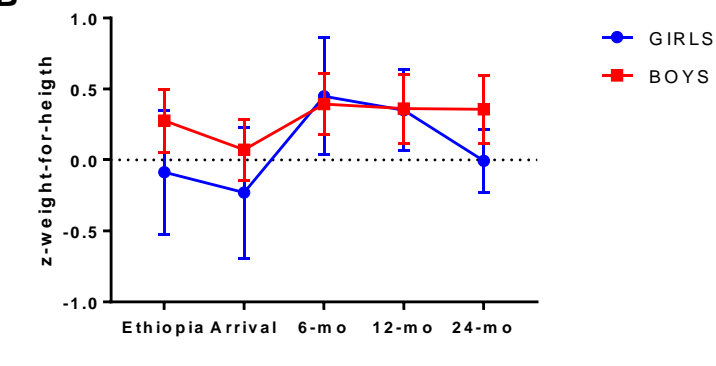

B

D

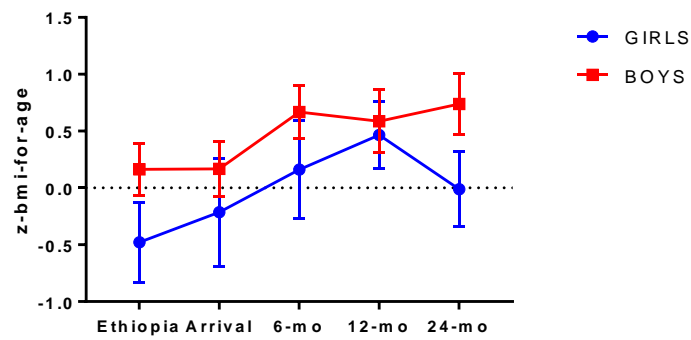

Figure 5. Comparison of the anthropometrical parameters evolution between Ethiopian girls and boys. Data indicate mean \pm sd. Baseline and follow-up z-scores for z-weight-forage (A), z-w eight-for-height (B), z-height-for-age (C) and z-bmi-for-age (D) are shown. The dotted line represents a $z$-score $=0$. Repeated measures ANOVA showed a statistically significant effect of time $(p<0.001)$ in all parameters. Sidak's multiple comparisons test was performed to compare data between Ethiopian girls and boys, but no statistical significant difference was observed.

\section{Mediterranean diet adherence and children growth}

Data derived from the KIDMED questionnaire showed that at 2 years of follow up, approximately $36 \%$ of the Ethiopian children showed adherence to the Mediterranean dietary pattern, while $64 \%$ showed a dietary pattern which lacked important nutrients for an optimal diet. Table 2 shows the degree of adherence to each of the KIDMED items. The adherence to the Mediterranean pattern was not associated with children's age $(p=0.255)$.

Overall, Ethiopian children showed good dietary habits since, most of them consumed fruit and vegetables regularly. However, we also found that a high proportion of children consumed sweets several times every day, and went to fast food restaurant more than once a week, which clearly deviates from the traditional Mediterranean diet (Table 2). 
Takes a fruit or fruit juice

Has a second fruit every day

Has fresh or cooked vegetables regularly once a day

Has fresh or cooked vegetables more than once a day

Consumes fish regularly (at least 2-3/week)

Goes $>1$ /week to a fast food restaurant (hamburguer)

Likes pulses and eats them $>1 /$ week

Consumes pasta or rice almost every day (5 or more per week)

Has cereals or grains (bread, etc) for breakfast

Consumes nuts regularly (at least 2-3/week)

Uses olive oil at home

Skips breakfast

Has a dairy product for breakfast (yoghurt, milk, etc)

Has commercially baked goods or pastries for breakfast

Takes two yoghurts and/or some cheese (40g) daily

Takes sweets and candy several times every day
237

Table 2. Degree of adherence to each of the KIDMED items at 2-years post-adoption. The degree of adherence refers to the percentage of subjects who score +1 on positive items (items 1-5, 7-11, 13 and 15) and subjects who score 0 on negative items (item 6, 12, 14 and 16). 0-3 points indicates a low adherence, a score of 4-7 describes average adherence, and a score of 8-12 reflects good adherence.

To evaluate the association between growth parameters and adherence to the Mediterranean dietary pattern, a correlation analysis was performed in the adopted children. Our data showed that there was a small but statistically significant positive correlation between the KIDMED score and increases in WHZ $(r=0.279, p=0.045)$ and HAZ ( $\mathrm{r}=0.385, p=0.004)$ after 6 months. In addition, the total HAZ increase after 2 years was positively correlated with the KIDMED score $(\mathrm{r}=0.374, p=0.006)$. These moderate correlations suggest that those children with higher KIDMED score showed higher increase especially in HAZ. 
The present study has prospectively evaluated the health status and the anthropometrical development of adopted Ethiopian children, from the moment they enter into the adoption process in Ethiopia, and over their first two years with their adoptive families in Spain. Our data showed that health problems decreased significantly two years after adoption. Adopted Ethiopian children showed progressive growth and were able to reach similar values when compared with the Spanish children. This growth was positively correlated with greater adherence to the Mediterranean diet.

Previous literature evaluating the health status of adopted Ethiopian children is limited. A study from 2016 evaluated the health status of these children upon arrival in Belgium [16], finding skin problems as the most prevalent, which is consistent with our findings. Martínez-Ortiz et al. (2017), observed similar health problems in this population, with more than a half of the children presenting skin disorders [17]. Another study conducted in the United States confirmed the high prevalence of skin disorders, although these authors also highlighted the presence of infectious disorders [6].

Outstandingly, although most health problems decreased, other dental or visual problems increased after the adoption process. It is important to note that several problems not detected in Ethiopian institutions, including cardiovascular or respiratory problems, appeared after two years in Spain. This situation may indicate that adopted children could have developed health problems after adoption, or most probably these problems were under-diagnosed at their home country. In fact, this data is consistent with a previous report performed on Eastern-European children where several neurological syndromes (e.g. fetal alcohol syndrome, Tourette syndrome, and attention deficit-hyperactivity disorder) were also underdiagnosed[18]. Nevertheless, other factors such as intrauterine growth retardation[19] or a developmental origin of disease owe to fetal re-programming cannot be excluded[20].

When evaluating the health problems of adopted children, one of the most determinant factors is the origin of the children. Previous works focused on international adoption have been conducted in Chinese and Eastern-European children, showing differences with Ethiopian children. For example, Miller and Hendrie described that the most common problem in Chinese children was elevated lead levels [21], which we didn't find in this research. Other work conducted in Romanian children 
283

adopted in the United States described recurrent intestinal and respiratory infections as frequent health conditions [22]. It is also important to note that medical information of internationally adopted children is limited and often outdated or inaccurate [23]. We advise that medical evaluation of these children should be carried out by multidisciplinary teams specialized in international adoption.

Regarding the anthropometrical development of the children adopted from Ethiopia, the first aspect to be highlighted is the low prevalence of thinness or wasting, which may indicate the absence of acute unfavorable conditions in these children. However, as described by the WHO, the prevalence of wasting is usually lower than $5 \%$, even in developing countries [24]. On the other hand, stunted growth was more prevalent, which may reflect a suboptimal health and/or nutritional condition. Our data are quite similar to those obtained in other populations. Miller and Hendrie described a prevalence of low z-scores for height of 39\% in children adopted from China[21]. Other studies on Eastern-European children reported a similar height but lower weight compared to the data we reported in this work [22,25]. Recent work of Fuglestad et al., with children from Eastern Europe, China and Ethiopia, have shown mean z-scores closer to those of our study [25]. In contrast, other work from Miller et al., in Ethiopian children, reported anthropometrical parameters that were mostly adequate [6].

Anthropometrical parameters of internationally adopted children are usually below the normal range. For this reason, one of the aims of the present study was to evaluate the prospective evolution of these children to determine if, in the long term, the growth parameters evolve similarly with those of the native-born population. Unfortunately, few studies have prospectively evaluated the evolution of adopted children, and most of them are focused on an endpoint analysis after a few years. We showed that the anthropometrical growth seems to be adequate at 2 years after adoption, which is consistent with the study of Rutter et al. conducted in Romanian children [22].A prospective study carried out by Cohen et al., in Chinese children described a constant increase in weight during the first 24 months of follow-up, as occurred in our population [26]. On the other hand, the study of Miller et al. also observed a significant catch-up growth after adoption, although not enough to reach normal values [6]. Since the work of Miller et al. did not subsequently evaluate the children, there is no evidence that the growth was sustained. 
One of the key determinants of children growth is dietary quality. Ethiopian diet is characterized by a low energy intake, mainly derived from grains, roots or tubers and little animal-sourced foods. Together with other factors (e.g., sanitation, hygiene, infectious diseases, etc.), a poor diet may be associated with a high rate of stunted growth [27]. The transition of these children towards a balanced diet can be challenging. However, our data indicated that most children had moderate or adequate adherence to the Mediterranean dietary pattern at 2 years of follow up, which was higher than expected and similar to those of native Mediterranean populations [28,29]. However, the study of Mariscal-Arcas et al., carried out in Spanish children, indicated greater adherence (48.6\%) that in the Ethiopian adopted children [30]. Overall, there seems to be a deterioration of the characteristics of healthy Mediterranean eating habits, which may explain the discrepancy between previous and more recent studies [31]. Consistent with our work, a study of Bibiloni et al. have reported a large number of children that skips breakfast (25\%) and go to fast food restaurants (17.6\%) [29]. We have shown a positive correlation between the KIDMED score and growth. It remains to be determined if the nutrients of the Mediterranean diet induce a better growth than other western-dietary patterns, but the few studies that have prospectively evaluated the growth of adopted children have only included other populations [22,32]. Overall, we have observed a greater anthropometric development than that described in other studies with a similar follow-up period [26].

It is also important to note that a healthy growth pattern should consider not only anthropometric parameters, but also neurocognitive variables. Therefore, an adequate growth does not depend exclusively on dietary factors, but also of other environmental and psychological factors. For example, a study conducted in Ethiopian children have shown that early exposure to maternal mental disorders was associated with impaired cognitive development. Attending to this work, several characteristics of the Ethiopian setting like poverty, interpersonal violence and infant undernutrition may decrease children development[33]. The study of Worku et al., in Ethiopian children have shown that children in extreme poverty performed worse in all the developmental domains compared to the reference Ethiopian children in a lower poverty group [34]. Therefore it seems reasonable to think that the adoption process can potentially improve not only the anthropometric parameters but also the neuropsychological development of these children. On the other hand, children development can involve a modification of 
anthropometric parameters but also improvements in cognitive performance, as shown in previous studies [35]. Our physical examination did not detect any cognitive delay, suggesting an adequate cognitive functionality in our population.

Several limitations in our work deserve consideration. Firstly, children's growth was determined solely through anthropometrical parameters, but other nutritional disorders (like anaemia or vitamin deficits) were not analysed. It is also important to note that the age provided by the Ethiopian adoption centre may be inaccurate, and may not correspond to the real biological age, which can influence the evaluation of growth. Furthermore, a large proportion of families with adopted children from Ethiopia (160 out of 336 children) declined to take part in the study, limiting the representativeness of our population. It is possible that the parents of children with severe or undiagnosed diseased decided not to take part in the study, so we cannot rule out a possible bias due to this circumstance. Finally, information on emotional, cognitive and psychological status was not considered in our analysis and could potentially explain some of the growth trajectories observed. It was not possible to assess the health status and the adherence to Mediterranean dietary pattern in the matched sample of children from Murcia (Spain).

\section{CONCLUSIONS}

The adoption process significantly improved the health status of adopted children from Ethiopia in Southern Spain. The number of clinical conditions was significantly reduced after 2 years post-adoption. While the baseline anthropometrical data indicate significant chronic undernutrition, our prospective evaluation of the children revealed a rapid growth development, which reached similar values to those of the native-born population in a period of 2 years. We also described the benefits of internationally-adopted children adapting to a balanced dietary pattern, such as the Mediterranean diet, since our data showed a positive association with adequate growth. Considering that the dietary habits of such young children depend almost exclusively on their parents, health education programs should be reinforced to improve the nutritional status of these children. Further studies are needed to fully evaluate the relevance of all potential factors involved in children development, including emotional, cognitive and 
psychological factors, in order to achieve an adequate health status, in all their dimensions, in these children.

Financial Support: This research received no specific grant from any funding agency, commercial or not-for-profit sectors.

Acknowledgements: The authors wish to thank Collaborative Entities of International Adoption - AMOFREM (Murcia, Spain) for its help in contacting the adoptive families of Ethiopian children.

\section{References}

[1] Cataldo F, Accomando S, Porcari V. Internationally adopted children: a new challenge for pediatricians. Minerva Pediatr 2006;58:55-62.

[2] Ampofo K. Infectious disease issues in adoption of young children. Curr Opin Pediatr 2013;25:78-87. doi:10.1097/MOP.0b013e32835c1357.

[3] Lesens O, Schmidt A, De Rancourt F, Poirier V, Labbe A, Laurichesse H, et al. Health Care Support Issues for Internationally Adopted Children: A Qualitative Approach to the Needs and Expectations of Families. PLoS One 2012;7:e31313. doi:10.1371/journal.pone.0031313.

[4] Piper BJ, Gray HM, Corbett SM, Birkett MA, Raber J. Executive Function and Mental Health in Adopted Children with a History of Recreational Drug Exposures. PLoS One 2014;9:e110459. doi:10.1371/journal.pone.0110459.

[5] Soares J, Barbosa-Ducharne M, Palacios J, Pacheco A. Adopted children's emotion regulation: The role of parental attitudes and communication about adoption. Psicothema 2017;29:49-54. doi:10.7334/psicothema2016.71.

[6] Miller LC, Tseng B, Tirella LG, Chan W, Feig E. Health of children adopted from Ethiopia. Matern Child Health J 2008;12:599-605. doi:10.1007/s10995007-0274-4.

[7] Mason P, Narad C. Long-Term Growth and Puberty Concerns in International Adoptees. Pediatr Clin North Am 2005;52:1351-68. doi:10.1016/j.pcl.2005.06.016.

[8] Robert M, Carceller A, Domken V, Ramos F, Dobrescu O, Simard M-N, et al. Physical and neurodevelopmental evaluation of children adopted from Eastern Europe. Can J Clin Pharmacol 2009;16:e432-40.

[9] Abera L, Dejene T, Laelago T. Prevalence of malnutrition and associated factors in children aged 6-59 months among rural dwellers of damot gale district, south Ethiopia: community based cross sectional study. Int $\mathbf{J}$ Equity Health 2017;16:111. doi:10.1186/s12939-017-0608-9. 
[10] Fresán U, Martínez-Gonzalez M-A, Sabaté J, Bes-Rastrollo M. The Mediterranean diet, an environmentally friendly option: evidence from the Seguimiento Universidad de Navarra (SUN) cohort. Public Health Nutr 2018:110. doi:10.1017/S1368980017003986.

[11] Iaccarino Idelson P, Scalfi L, Valerio G. Adherence to the Mediterranean Diet in children and adolescents: A systematic review. Nutr Metab Cardiovasc Dis 2017;27:283-99. doi:10.1016/j.numecd.2017.01.002.

[12] M DO. The new WHO child growth standards. Paediatr Croat Suppl 2008;52:137. doi:10.4067/S0370-41062009000400012.

[13] WHO |WHO Anthro (version 3.2.2, January 2011) and macros. WHO 2017.

[14] Serra-Majem L, Ribas L, Ngo J, Ortega RM, García A, Pérez-Rodrigo C, et al. Food, youth and the Mediterranean diet in Spain. Development of KIDMED, Mediterranean Diet Quality Index in children and adolescents. Public Health Nutr 2004;7:931-5.

[15] Štefan L, Prosoli R, Juranko D, Čule M, Milinović I, Novak D, et al. The Reliability of the Mediterranean Diet Quality Index (KIDMED) Questionnaire. Nutrients 2017;9. doi:10.3390/nu9040419.

[16] Van Kesteren L, Wojciechowski M. International adoption from Ethiopia: An overview of the health status at arrival in Belgium. Acta Clin Belgica Int J Clin Lab Med 2017;72:300-5. doi:10.1080/17843286.2016.1258178.

[17] Ortiz AM, Pinilla ND, Wudineh M. Adopción internacional de Etiopía en un período de 5 años International adoption from Ethiopia in a 5-year period Resumen Introducción Método Resultados Conclusiones Palabras clave Keywords 2017:1-9.

[18] Fernández-Mayoralas DM, Fernández-Jaén A, Muñoz-Jareño N, Calleja Pérez B, Arroyo-González R. Fetal Alcohol Syndrome, Tourette Syndrome, and Hyperactivity in Nine Adopted Children. Pediatr Neurol 2010;43:110-6. doi:10.1016/j.pediatrneurol.2010.03.008.

[19] Chatmethakul T, Roghair RD. Risk of hypertension following perinatal adversity: IUGR and prematurity. J Endocrinol 2019;242.

[20] Zou K, Ding G, Huang H. Advances in research into gamete and embryo-fetal origins of adult diseases. Sci China Life Sci 2019;62:360-8. doi:10.1007/s11427018-9427-4.

[21] Miller LC, Hendrie NW. Health of Children Adopted From China. Pediatrics 2000;105:e76-e76. doi:10.1542/peds.105.6.e76.

[22] Rutter M, Andersen-Wood L, Beckett C, Bredenkamp D, Castle J, Dunn J, et al. Developmental catch-up, and deficit, following adoption after severe global early privation. J Child Psychol Psychiatry Allied Discip 1998;39:465-76. doi:10.1017/S0021963098002236.

[23] Miller BS, Kroupina MG, Mason P, Iverson SL, Narad C, Himes JH, et al. Determinants of Catch-Up Growth in International Adoptees from Eastern Europe. Int J Pediatr Endocrinol 2010;2010:1-8. doi:10.1155/2010/107252. 
[24] Who. Global Database on Child Growth and Malnutrition. Chem ... 2010;2:2-3. doi:10.1093/tandt/11.7.180.

[25] Fuglestad AJ, Kroupina MG, Johnson DE, Georgieff MK. Micronutrient status and neurodevelopment in internationally adopted children. Acta Paediatr Int J Paediatr 2016;105:e67-76. doi:10.1111/apa.13234.

[26] Cohen NJ, Lojkasek M, Zadeh ZY, Pugliese M, Kiefer H. Children adopted from China: A prospective study of their growth and development. J Child Psychol Psychiatry Allied Discip 2008;49:458-68. doi:10.1111/j.14697610.2007.01853.x.

[27] Gashu D, Stoecker BJ, Adish A, Haki GD, Bougma K, Marquis GS. Ethiopian pre-school children consuming a predominantly unrefined plant-based diet have low prevalence of iron-deficiency anaemia. Public Health Nutr 2016;19:1834-41. doi:10.1017/S1368980015003626.

[28] Štefan L, Prosoli R, Juranko D, Čule M, Milinović I, Novak D, et al. The Reliability of the Mediterranean Diet Quality Index (KIDMED) Questionnaire. Nutrients 2017;9. doi:10.3390/nu9040419.

[29] del Mar Bibiloni M, Pons A, Tur JA. Compliance with the Mediterranean Diet Quality Index (KIDMED) among Balearic Islands' Adolescents and Its Association with Socioeconomic, Anthropometric and Lifestyle Factors. Ann Nutr Metab 2016;68:42-50. doi:10.1159/000442302.

[30] Mariscal-Arcas M, Rivas A, Velasco J, Ortega M, Caballero AM, Olea-Serrano F. Evaluation of the Mediterranean Diet Quality Index (KIDMED) in children and adolescents in Southern Spain. Public Health Nutr 2009;12:1408. doi:10.1017/S1368980008004126.

[31] Rizza W, De Gara L, Antonelli Incalzi R, Pedone C. Prototypical versus contemporary Mediterranean Diet. Clin Nutr ESPEN 2016;15:44-8. doi:10.1016/j.clnesp.2016.06.007.

[32] Fuglestad AJ, Kroupina MG, Johnson DE, Georgieff MK. Micronutrient status and neurodevelopment in internationally adopted children. Acta Paediatr Int J Paediatr 2016;105:e67-76. doi:10.1111/apa.13234.

[33] Servili C, Medhin G, Hanlon C, Tomlinson M, Worku B, Baheretibeb Y, et al. Maternal common mental disorders and infant development in Ethiopia: The PMaMiE Birth Cohort. BMC Public Health 2010;10. doi:10.1186/1471-2458-10693.

[34] Worku BN, Abessa TG, Wondafrash M, Vanvuchelen M, Bruckers L, Kolsteren $\mathrm{P}$, et al. The relationship of undernutrition/psychosocial factors and developmental outcomes of children in extreme poverty in Ethiopia. BMC Pediatr 2018;18:1-9. doi:10.1186/s12887-018-1009-y.

[35] Park H, Bothe D, Holsinger E, Kirchner HL, Olness K, Mandalakas A. The impact of nutritional status and longitudinal recovery of motor and cognitive milestones in internationally adopted children. Int J Environ Res Public Health 2011;8:105-16. doi:10.3390/ijerph8010105. 


\section{${ }^{*}$ Conflict of Interest}

\section{Declaration of interests}

$\bigotimes$ The authors declare that they have no known competing financial interests or personal relationships that could have appeared to influence the work reported in this paper.

$\square$ The authors declare the following financial interests/personal relationships which may be considered as potential competing interests:

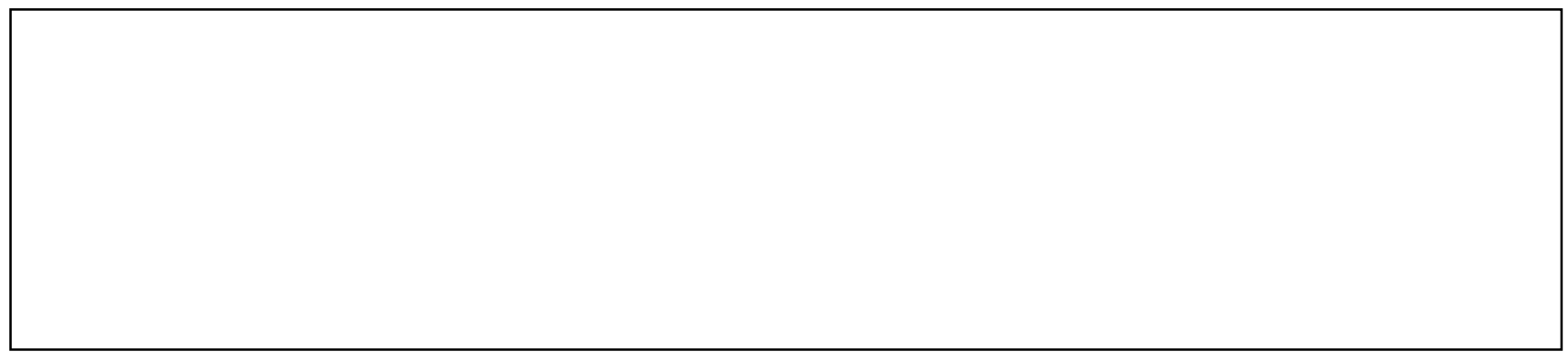




\section{REVIEWER SUGGESTIONS}

1. Fátima Pérez de Heredia. F.PerezDeHerediaBenedicte@ljmu.ac.uk. Liverpool John Moores University, UK.

2. Carol Mest. carol.mest@ desales.edu. DeSales University, USA.

3. Miguel Reyes. miguel.reyes@usach.cl. Universidad Autónoma de Chile, Chile.

4. Javier Tébar Massó. jtebar2@gmail.com. Endocrinology Service, Arrixaca Hospita, Murcia, Spain.

5. Luis Moreno Aliaga. lmoreno@unizar.es. Universidad de Zaragoza, Spain. 